RESEARCH ARTICLE

\title{
Caseous Rhinosinusitis: Fungal or Bacterial Ball?
}

\author{
Natalia V Boiko
}

\section{Abstract}

Background: The fungal ball is a common type of fungal rhinosinusitis. The fungal ball appears to be unstructured caseous masses revealed during the surgical treatment.

Aim and objective: To analyze the contents of the paranasal sinuses in 168 patients with the clinically suspected fungal ball who had undergone functional endoscopic sinus surgery (FESS).

Study design: A total of 168 patients aged between 19 and 63 with fungal ball clinical diagnosis admitted to the ENT Department of Rostov State Medical University, Russia, were involved in the study between January 2009 and July 2018.

Materials and methods: Tissue samples obtained from the affected sinuses underwent microscopic, microbiological, and histopathological examination.

Results: Fungi presence was confirmed in 148 out of 168 patients. In 20 cases, the presence of fungi in the caseous masses, obtained during the operation, was not revealed by any diagnostic method, and in 19 out of 20 patients, different types of bacteria were detected in diagnostically significant titers, most often Bacteroides spp.- -in five patients, Staphylococcus spp.-in five patients, Pseudomonas aeruginosa-in three patients, two cases of Klebsiella pneumoniae, and two cases of Actinomyces spp. To characterize such findings, the term "bacterial ball" by analogy with the "fungal ball" was earlier suggested.

Conclusion: Caseous masses found during sinus surgery is often considered to be a clinical diagnosis validation. Meanwhile, the said caseous masses may not only manifest a fungal disease, but also bacterial colonization, which determines the postoperative treatment.

Keywords: Caseous sinusitis, Culture, Fungal ball, Paranasal sinuses.

Otorhinolaryngology Clinics: An International Journal (2019): 10.5005/jp-journals-10003-1342

\section{INTRODUCTION}

The problem of fungal diseases in the ENT practice has been holding relevance over several years. The noted growth of fungal rhinosinusitis ${ }^{1,2}$ is a consequence of the increased risk factors of its development, such as broad-spectrum antibiotic application, iatrogenic secondary immunodeficiency resulting from long-term treatment with glucocorticosteroid and cytostatics, inadequate endodontic treatment.

Fungal rhinosinusitis is categorized as either noninvasive or invasive. The noninvasive forms, which are generally manifested in immunocompetent patients, include localized fungal colonization, fungal ball, and allergic fungal rhinosinusitis. Invasive forms, usually seen in immunocompromised patients, include acute and chronic invasive fungal rhinosinusitis. ${ }^{1,3}$ In addition, there is also a type of granulomatous invasive fungal rhinosinusitis ${ }^{4}$ that affects immunocompetent patients almost exclusively in the Middle East, Africa, and India. ${ }^{5-7}$

It is often rather difficult to detect fungal balls as the clinical symptoms of the disease are non-specific ${ }^{8,9}$ and the patients typically undergo prolonged treatment in outpatient departments for "chronic rhinosinusitis".

The basic method in fungal ball pre-surgery detection is a noncontrast computerized tomography (CT). A fungal ball typically appears as hyperattenuating on noncontrast CT scan with intrasinus calcification (69-77\%) ${ }^{10}$ and reactive bony walls sclerosis. ${ }^{11}$

The treatment of choice for the fungal ball is endoscopic sinus surgery. ${ }^{12}$ Surgery reveals the sinuses' content being unstructured caseous masses. As the clinical practice shows, this content is not always a fungal ball. ${ }^{13,14}$

The objective of the study is to analyze the results of microscopy, microbiological, and histopathological examinations
Department of ENT, Rostov State Medical University, Rostov-on-Don, Rostov Region, Russian Federation

Corresponding Author: Natalia V Boiko, Department of ENT, Rostov State Medical University, Rostov-on-Don, Rostov Region, Russian Federation, Phone: +79034334113, e-mail: nvboiko@gmail.com

How to cite this article: Boiko NV. Caseous Rhinosinusitis: Fungal or Bacterial Ball? Int J Otorhinolaryngol Clin 2019;11(3):64-66.

Source of support: Nil

Conflict of interest: None

of the contents of the paranasal sinuses in patients with a clinically suspected fungal ball.

\section{Materials and Methods Study Design}

A prospective cohort study was undertaken between January 2009 and July 2018 at the Department of Otorhinolaryngology of Rostov State Medical University, Russian Federation. The study involved 168 patients aged between 19 and 63 with a clinically suspected fungal ball.

\section{Criteria for Inclusion}

Persistent symptoms of chronic rhinosinusitis, heterogeneous opacity within sinus on noncontrast CT scans, unstructured caseous masses found during endoscopic sinus surgery.

The sinus content was collected intraoperatively into a sterile container Multipurpose Clinical Sample Collector PP (Hi-Media, India) and within 30 minutes transported to the laboratory. Tissue samples for histopathological examination were put into $10 \%$ 
formalin. Primary microscopy was carried out in Gram's, calcofluor white, hematoxylin and eosin (H\&E) staining. Calcofluor is a fluorochrome stain having affinity to the fungal cell wall chitin, it is absorbed by the mycelium and shows a typical glow if examined by a fluorescent microscope.

This was followed by bacteriological and histopathological examinations. Bacteriological examination of the sinus content was carried out with special and elective nutritional media for aerobic, anaerobic bacteria, and fungi under temperatures of 25 and $37^{\circ} \mathrm{C}$ and examined for 28 days.

All histological sections were stained by H\&E, Periodic acidSchiff (PAS), and Gomori Methenamine Silver (GMS) stains.

This study was conducted in accordance with the declaration of Helsinki. Ethical approval was taken from the Ethical Committee of Rostov State Medical University.

\section{Results}

The total number of patients who underwent functional endoscopic sinus surgery (FESS) during the analyzed period was 1,449. Based on the pre-surgery CT data and the appearance of the sinus pathological content during surgery, 168 patients were diagnosed as a fungal ball.

A definitive diagnosis of the fungal ball was confirmed by microscopic, cultural, and histopathological examinations in 148 out of 168 patients ( $10.2 \%$ of the total number of operated patients). Out of 148 patients, 145 (86.3\%) were found to be positive by histopathology, 129 (76.8\%)—by direct microscopy, 153 (91.1\%)—by microscopy of samples stained by calcofluor, and only 30 (17.8\%) by culture. By using aerobic and anaerobic culture, bacterial coinfection was revealed in 109 out of 148 patients (73.6\%) with a confirmed fungal ball.

In 20 cases (1.38\% of the total number of patients) in the caseous masses obtained during surgery, no fungi were revealed by microscopic, or cultural, or histological examinations; yet 19 patients revealed bacteria in diagnostically significant titers (14 patients showed aerobic bacteria, 1 showed anaerobic bacteria, and 4 showed mixed aerobic-anaerobic bacteria), and 1 patient did not reveal any microorganisms. In 15 cases, the fungal ball was localized in the maxillary sinus, in 5 cases it was the sphenoid sinus. The results of the microbiological study of the sinus content obtained during FESS are represented in Table 1.

Anaerobic bacteria were represented by Bacteroides spp. detected in four patients in aerobic-anaerobic associations with Staphylococcus aureus (two cases), with Klebsiella pneumoniae, Escherichia coli, and in a monovariant (one case).

Thus, most frequently detected from out of the caseous masses obtained during surgery, were Bacteroides spp., staphylococci spp. (five cases each), Pseudomonas aeruginosa (3 patients), $K$. pneumoniae, and Actinomyces spp. (two cases each).

\section{Discussion}

The fungal ball is characterized by the accumulation of the dense conglomeration of fungal hyphae in the sinus cavity. ${ }^{3}$ But unstructured caseous masses found during sinus surgery are not a doubtless sign of a fungal ball. This was first noted by Braun et al. ${ }^{13}$ The authors proposed the term caseous sinusitis to denote such cases. Later, in 2016, Kim et al. ${ }^{14}$ described unusual findings they had made during sinuses surgery in two patients. They presented

\begin{tabular}{lc}
\hline Microorganisms, content (CFU/mL) & $\begin{array}{l}\text { No. of } \\
\text { patients }\end{array}$ \\
\hline Pseudomonas aeruginosa, $10^{7}$ & 3 \\
Staphylococcus aureus, $10^{5}$ & 1 \\
Staphylococcus aureus, $10^{5}+$ Bacteroides spp., $10^{6}$ & 2 \\
Staphylococcus epidermidis, $10^{7}$ & 2 \\
Klebsiella pneumoniae, $10^{5}$ & 1 \\
Klebsiella pneumoniae, $10^{6}+$ Bacteroides spp., $10^{5}$ & 1 \\
Actinomyces spp., $10^{5}$ & 2 \\
Klebsiella oxytoca, $10^{4}+$ Staphylococcus aureus, $10^{5}+$ & 1 \\
Streptococcus spp., $10^{4}$ & \\
Pseudomonas aeruginosa, $10^{6}+$ Enterococcus faecalis, $10^{6}$ & 1 \\
Escherichia coli, $10^{6}$, Bacteroides spp., $10^{5}$ & 1 \\
Haemophilus spp., $10^{6}$ & 1 \\
Enterobacter cloacae, $10^{6}+$ Morganella morganii, $10^{7}$ & 1 \\
Enterobacter aerogenes, $10^{5}+$ Stenotrophomonas & 1 \\
maltophilia, $10^{5}$ & \\
Bacteroides spp., $10^{5}$ & 1 \\
Absence of growth of microorganisms & 1 \\
Total & 20 \\
\hline
\end{tabular}

two cases of intractable rhinosinusitis with "pseudomonas-induced macroscopic biofilms (bioballs)" of the maxillary sinuses. ${ }^{14}$

In 2018, the same authors ${ }^{15}$ published an article describing six cases of similar findings in the maxillary sinuses and suggested to name this amorphous material, which reveals bacteria, a "bacterial ball" by analogy to the term a "fungal ball". A bacterial ball might be a new kind of organized community of bacteria raising its survivability, as supposed by Kim et al. The presence of bacteria in caseous masses was only detected by microscopic study, yet their identification was not carried out.

Chronic rhinosinusitis is associated with the imbalance of the sinus bacterial microbiome. ${ }^{16}$ Most bacteria in vivo exist rather as biofilms than in a planktonic form. ${ }^{17}$ The prevalence of biofilm in patients with chronic rhinosinusitis has been reported in a wide range of spectrum ${ }^{18,19}$ and is a mechanism of the formation of microorganisms' antibacterial resistance.

We have accumulated 20 cases of chronic rhinosinusitis with sinus content resembling fungal balls, yet being a manifestation of sinus bacterial colonization and identified these microorganisms. The sinus structureless masses may be gigantic biofilms because the microorganisms found by us-P. aeruginosa, S. aureus, K. pneumoniae-show enhanced capability toward biofilm formation which, even within one taxon, depends on the expression of corresponding genes and correlates with the virulence degree. ${ }^{20}$

Our study had its limitations. We had no opportunity for biofilm determination, which might help to establish the nature of the fungal ball.

\section{Conclusion}

A fungal ball is a common form of noninvasive mycosis of the paranasal sinuses. In our study, fungal balls have been revealed during the surgical treatment of chronic rhinosinusitis in $10.2 \%$ of cases. 
A fungal ball is usually suspected radiologically by $\mathrm{CN}$ scan since the clinical manifestations are non-specific. Detection of caseous masses during sinus surgery is often considered to be a clinical diagnosis confirmation. Meanwhile, these masses may not only manifest a sinus fungal disease, but also bacterial colonization, which determines the choice of postoperative patient management. Late years' publications describe few instances of such findings and propose a new term "bacterial ball" to denote a pathological process of this kind. This may be a new form of an organized community of bacteria, although one can neither exclude other mechanisms of formation of such space-occupying lesion. To clarify the nature of the bacterial ball we need further investigation.

\section{Clinical Relevance}

Our study has shown a necessity to investigate the sinus content even in those cases where the clinical diagnosis "fungal ball" seems to be in no doubt. In cases where the diagnosis has been confirmed, the fungal ball completely removed during surgery and the drainage from the affected sinus re-established, there is no need for any drug therapy during the postoperative period. A bacterial ball found in the sinus demands antibacterial therapy after the surgery.

\section{REFERENCES}

1. Denning DW, Chakrabarti A. Pulmonary and sinus fungal diseases in non-immunocompromised patients. Lancet Infect Dis 2017;17(11):357-366. DOI: 10.1016/S1473-3099(17)30309-2.

2. Singh AK, Gupta P, Verma N, et al. Fungal rhinosinusitis: microbiological and histopathological perspective. J Clin Diagn Res 2017;11(7):DC10 DC12. DOI: 10.7860/JCDR/2017/25842.10167.

3. Chakrabarti A, Denning DW, Ferguson BJ, et al. Fungal rhinosinusitis: a categorization and definitional schema addressing current controversies. Laryngoscope 2009;119(9):1809-1818. DOI: 10.1002/ lary. 20520.

4. Fokkens WJ, Ebbens F, van Drunen CM. Fungus: a role in pathophysiology of chronic rhinosinusitis, disease modifier, a treatment target, or no role at all? Immunol Allergy Clin North Am 2009;29(4):677-688. DOI: 10.1016/j.iac.2009.07.002.

5. Panda NK, Sharma SC, Chakrabarti A, et al. Paranasal sinus mycoses in north India. Mycoses 1998;41(7-8):281-286. DOI: 10.1111/j.14390507.1998.tb00339.x.

6. Michael RC, Michael JS, Ashbee RH, et al. Mycological profile of fungal sinusitis: an audit of specimens over a 7-year period in a tertiary care hospital in Tamil Nadu. Indian J Pathol Microbiol 2008;51(4):493-496. DOI: 10.4103/0377-4929.43738.
7. Montone KT, Livolsi VA, Feldman MD, et al. Fungal rhinosinusitis: a retrospective microbiologic and pathologic review of 400 patients at a single university medical center. Int J Otolaryngol 2012;2012:684835. DOI: 10.1155/2012/684835.

8. Yoon $\mathrm{YH}, \mathrm{Xu}$ J, Park SK, et al. A retrospective analysis of 538 sinonasal fungus ball cases treated at a single tertiary medical center in Korea (1996-2015). Int Forum Allergy Rhinol 2017;7(11):1070-1075. DOI: 10.1002/alr.22007.

9. Aït-Mansour A, Pezzettigotta S, Genty E, et al. Evaluation of the prevalence and specificities of asymptomatic paranasal sinus aspergillosis: retrospective study of 59 cases. European Annals of Otorhinolaryngol, Head and Neck Diseases 2015;132:19-23.

10. Seo Y-J, Kim J, Kim K, et al. Radiologic characteristics of sinonasal fungus ball: an analysis of 119 cases. Acta Radiol 2011;52(7):790-795. DOI: 10.1258/ar.2011.110021.

11. Khattar VS, Hathiram BT. Radiologic appearances in fungal rhinosinusitis. Otorhinolaryngology Clinics: An International Journal 2009;1(1):15-23. DOI: 10.5005/jp-journals-10003-1002.

12. Hathiram BT, Khattar VS. Surgical management of fungal rhinosinusitis. Otorhinolaryngology Clinics: An International Journal 2009;1(1):69-75. DOI: 10.5005/jp-journals-10003-1010.

13. Braun JJ, Bourjat $P$, Gentine A, et al. Caseous sinusitis. clinical, $x$-ray computed, surgical, histopathological, biological, biochemical and myco-bacteriological aspects. Apropos of 33 cases. Ann Otolaryngol Chir Cervicofac 1997;114(4):105-115.

14. Kim DK, Lee DW, Pyo JY, et al. Bioballs causing asymptomatic or recurrent acute rhinosinusitis: two cases. J Rhinol 2016;23(1):55-59. DOI: 10.18787/jr.2016.23.1.55.

15. Kim DK, Wi YC, Shin SJ, et al. Bacterial ball as an unusual finding in patients with chronic rhinosinusitis. Clin Exp Otorhinolaryngol 2018;11(1):40-45. DOI: 10.21053/ceo.2017.00332.

16. Bordin A, Sidjabat HE, Cotrell K, et al. Chronic rhinosinusitis: a microbiome in dysbiosis and the search for alternative treatment options. Microbiology Australia 2016;37(3):149-152. DOI: 10.1071/ MA16051.

17. Al-Mutairi D, Kilty SJ. Bacterial biofilms and the pathophysiology of chronic rhinosinusitis. Curr Opin Allergy Clin Immunol 2011;11(1):1823. DOI: $10.1097 / \mathrm{ACl} .0 \mathrm{~b} 013 \mathrm{e} 3283423376$.

18. Psaltis AJ, Ha KR, Beule AG, et al. Confocal scanning laser microscopy evidence of biofilms in patients with chronic rhinosinusitis. Laryngoscope 2007;117(7):1302-1306. DOI: 10.1097/ MLG.0b013e31806009b0.

19. Tatar EC, Tatar I, Öcal B, et al. Prevalence of biofilms and their response to medical treatment in chronic rhinosinusitis without polyps. Otolaryngol Head Neck Surg 2012;146(4):669-675. DOI: 10.1177/0194599811434101.

20. Marsh PD. Dental plaque as a biofilm and a microbial community implications for health and disease. BMC Oral Health 2006;6(Suppl 1):14-20. DOI: 10.1186/1472-6831-6-S1-S14. 